\title{
State of the "Fine" art in the age of artificial intelligence
}

\author{
Eizaburo Ohno ${ }^{1}$
}

() The Japan Society of Ultrasonics in Medicine 2019

I went to a gallery the other day to enjoy some paintings. The gallery was packed with people, partly because word had spread that a painting by a famous foreign impressionist painter was on display. It took me an hour to make my way through the crowd and finally reach the portrait by Renoir that I had come to see. Despite the fact that it is a world famous painting and I had seen photographs of it many times in books and on the Internet, I was struck and very moved by the beauty of the actual painting. Several days later, I was browsing through an art catalog and found detailed information about the painting I had seen. It included information like the family background of the woman in the painting, when it was painted, the techniques used to paint it, and even a list of the collectors who had owned it over the years. Knowing all this information might help us more deeply understand the painting, but I personally felt like my passion had been slightly dampened. Academic descriptions aimed at elucidating the beauty of a work of art and the sensibility to embrace beauty do not necessarily go together.

The expression "state of the art" refers to cutting-edge technology in the field of science. In the case of both works of art and diagnostic imaging used in medicine, expressing the characteristics of an image in words is an important means of giving many people a shared awareness of an image, and it serves to make information objective. On the other hand, I feel as if attaching too much importance to verbal descriptions of "state of the art" will result in all paintings and images being reduced to cold entities that can be understood in an automatic manner. Use of artificial intelligence (AI) technology, which has been showing rapid progress in recent years, is expected to become commonplace in the world of medicine in the very near future. In the area of diagnostic imaging, in particular, the accuracy and speed of AI-based diagnosis are expected to eclipse those of humans by far. In the case of ultrasound imaging, as well, complex algorithms will likely be used in the future to explain subtle characteristics that cannot be verbalized by physicians.

Control of image quality and objectivization of imaging findings are the most crucial aspects of diagnostic imaging. Nevertheless, I think it is important that we do not become too attached to cold guides and manuals and that we do not lose our human sensibility that allows us to experience "beauty" in an intuitive and passionate way.

Personally, it is my strong hope that I will be a doctor who can understand the "beauty" of ultrasound images in a scientific and passionate manner.

Publisher's Note Springer Nature remains neutral with regard to jurisdictional claims in published maps and institutional affiliations.
Eizaburo Ohno

eono@med.nagoya-u.ac.jp

1 Department of Gastroenterology and Hepatology, Nagoya University Graduate School of Medicine, Aichi, Japan 